\title{
SITUACIÓN ACTUAL DEL TABAQUISMO ENTRE LOS TRABAJADORES DE CORREOS-ESPAÑA TRAS LA LEY 28/2005
}

\author{
PRESENT TOBACCO SITUATION BETWEEN THE CORREOS-ESPAÑA \\ EMPLOYEES AFTER THE LAW 28/2005
}

\author{
M.T. VICENTE-HERRERO ${ }^{(1)}$, A.A LÓPEZ-GONZÁLEZ(2), PEPA PONT-MARTÍNEZ ${ }^{(3)}$, \\ C. SANTAMARÍA-NAVARRO ${ }^{(4)}$, SUSANA DE PAZ MERCHAN( ${ }^{(5)}$, ROSA RODRÍGUEZ-FERNÁNDEZ $^{(6)}$
}

(1) Área de Salud Laboral. Correos. Valencia. España.

(2) Servicio de Prevención de GESMA. Palma de Mallorca. España.

(3) Dirección General de Salud Pública. Conselleria de Sanitat .Valencia. España.

(4) Instituto de Matemática Multidisciplinar. Universidad Politécnica de Valencia. España.

(5) Área de Salud Laboral. Correos. Madrid. España.

(6) Área de Salud Laboral. Correos. Cádiz. España.

\section{RESUMEN}

Objetivos: Conocer la prevalencia de tabaquismo entre nuestros trabajadores, el historial tabáquico y las diferencias de consumo por edad, sexo, puesto de trabajo y variaciones geográficas en el personal de Correos.

Material y métodos: cuestionario elaborado "ad hoc" a 1432 trabajadores de Correos en 5 provincias españolas, sin selección previa. La captación fue durante la realización de los exámenes de vigilancia periódica de la salud. Participación anónima, voluntaria y consentimiento previo para la utilización epidemiológica de los datos estadísticos, analizados con el programa SPSS v 14.

Resultados: Edad media 44,09 años. Sexo: 56\% hombres, $44 \%$ mujeres. Colectivo mayoritario carteros/as. No fumadores $39,1 \%$, Fumadores diarios 29,9\%, Fumadores ocasionales 5,2\%, Ex fumadores $29,7 \%$. No variaciones geográficas ni por sexo en los resultados obtenidos. Más fumadores activos en puestos de trabajo con mayor cualificación profesional. Mayor numero de fumadores activos entre los 36-50 años.

Conclusiones: Tasa de participación elevada, superior al 85\%. La edad de los participantes coincidente con la edad media de nuestra población laboral. En Correos, predomina el personal nofumador, aunque persiste una elevada cifra de fumadores/as. $35 \%$, por encima de las tasas de consumo en la población general $(31 \%$ ENS 2003 y el 30\% según datos provisionales de la ENS 2006). No influye en el habito tabáquico la localización geográfica, ni el sexo, aunque si hay diferencias significativas en función del puesto de trabajo y la edad.

Palabras clave: Tabaco empresa, tabaco trabajadores, ley del tabaco empresa, Ley del tabaco trabajadores.

(Med Segur Trab 2008; 54 (213):87-95)

\section{ABSTRACT}

Objectives: To know about the prevailing tobacco habit among our workers; the tobacco addiction records and the consumption differences by ages, males/females, work positions and geographical variations by the Correos employees.

Materials and methods used: A suitable elaborated anonymous questionnaire carried out to 1432 Correos workers in 5 different Spanish provinces, without a previous selection. They were asked to complete the questionnaire during their regular health controls and medical examinations. Voluntary anonymous participation and previous consent to use the statistical epidemic data information, analyzed with the program SPSS v 14.

Results: Average age 44.09 years. Sexes: $56 \%$ males, $44 \%$ females. Main collective of participators, postmen/women. Non smokers $39.1 \%$, daily smokers $29.9 \%$, occasional smokers $5.2 \%$, former smokers $29.7 \%$. The results did not show geographical or sexes variations. There were more active smokers in work positions with a better professional qualification. A major number of active smokers in the range of age from 36 to 50 years.

Conclusions: An elevated rate of participation, more than $85 \%$. The age of the participants coincide with the average age of our labour population. In Correos predominate over the non smokers employees, even though we have an elevated number of smokers among male and female. $35 \%$ over the population consumption rate $(31 \%$ ENS in 2003 and 30\% ENS provisional rate for 2006). The tobacco habit has not any influence with the geographical area or sexes of the participants, but has significant differences depending on the working positions and ages.

Key words: Tobacco employees, tobacco company, tobacco Law Company. Employees tobacco law..

\section{Correspondencia:}

Dra. Ma Teófila Vicente Herrero.

Área de Salud Laboral - Correos.

Plaza del Ayuntamiento, 24-2

46002 Valencia. España

Teléfono: 963102752/Fax: 963940500

e-mail: teovicente@ono.com

(Med Segur Trab 2008; 54 (213):87-95) 


\section{INTRODUCCIÓN}

El consumo de tabaco en España, al igual que en el resto de países y a pesar de los cambios sociales de los últimos años, todavía está socialmente aceptado, incluso en el ámbito laboral, pese a su catalogación como droga adictiva por sus características de tolerancia, dependencia física y psicológica. La dependencia al tabaco esta reconocida como un trastorno mental y del comportamiento tanto por la OMS (ICD-10), como por el manual de la asociación americana de psiquiatría (DSM-IV) (1).

Según los datos recogidos en el avance de la encuesta de salud 2006-2007 del ministerio de sanidad y consumo, publicados en Marzo del presente año 2007 (2), la cifra de fumadores en España, actualmente se situaría en el $29,95 \%$, frente a un $48,92 \%$ de personas que nunca han fumado y un $21,13 \%$ de ex fumadores. Sigue existiendo una clara predominancia de hombres sobre mujeres entre los fumadores y ex fumadores, mientras que las mujeres se imponen entre las personas que nunca han fumado. En cuanto a la edad, en los hombres, el mayor número de fumadores se encuentra en el bloque de edad de 25-44 años, mientras que en las mujeres la mayor proporción de fumadoras, se sitúa entre los 16-44 años, en las etapas fértiles de su vida, según resultados de la encuesta nacional de salud en España-2006.

Pero es la morbilidad y mortalidad asociada al consumo de tabaco (3), su coste socio- sanitario y laboral (4) y el ser un factor de riesgo sobre el que puede actuarse en prevención, lo que ha hecho que se haya actuado con especial interés sobre el tabaco desde el mundo laboral y especialmente tras la entrada en vigor en España de la ley del tabaco 28/2005 (5), cuando la actuación sobre el tabaquismo, incorpora junto a las actitudes preventivas, una dimensión mucho más intervencionista del personal de medicina y enfermería del trabajo, aumentando las actividades de apoyo a la deshabituación, apoyando la prohibición del consumo de tabaco en el ámbito laboral. Con el objetivo de desarrollar estas acciones, se han elaborado Guías prácticas de actuación integral frente al tabaquismo desde los servicios de prevención de las empresas y desde las instituciones sanitarias tanto autonómicas como centrales-(6) (7) (8) (9).

La prevalencia de consumo de tabaco en el grupo de 25 a 44 años, donde se encuentra una buena parte de la población activa, se estima en el $40 \%$ y si consideramos el intervalo de 16 a 65 años (población activa a cargo de la medicina del trabajo), la prevalencia de consumo es del 36,56\% (ENS 2003) (10)

Correos, puede suponer una referencia en el mundo del trabajo en España, ya que actualmente, es la empresa estatal con mayor número de personas trabajadoras del territorio español (65.000 trabajadores/as), lo que nos permite realizar estudios sobre muestras de población, lo suficientemente amplias, como para que los resultados alcanzados puedan ser referencia posterior para otros colectivos de población ocupada en centros de trabajo públicos o privados.

Nos ha interesado como objetivo principal de este estudio, el conocer las características de nuestro personal trabajador en Correos, respecto al consumo de tabaco, con el objeto de plantear una posterior intervención desde los servicios médicos de la empresa. Como objetivos específicos, hemos querido identificar la prevalencia e historial tabáquico de nuestros trabajadores/as y establecer las posibles diferencias estadísticas en el consumo en función de la edad, sexo, puesto de trabajo y variaciones geográficas de nuestros trabajadores/as.

\section{MATERIAL Y MÉTODOS}

En las cinco provincias Españolas participantes (Cádiz, Gerona, Madrid, Castellón y Valencia), se realizó una encuesta dirigida al personal de Correos, que había solicitado previamente reconocimiento periódico de vigilancia de la salud durante la campaña 2006-2007. Se utilizó para ello una encuesta ya validada (encuesta europea de hospitales sin humo), que fue adaptada a las características y puestos de trabajo de nuestra empresa. La participación, fue voluntaria y anónima. Se solicitó el consentimiento por escrito, para la explotación epidemiológica y posterior publicación de los resultados obtenidos y se informó a los Comités Provinciales de seguridad y salud, tanto al comienzo de la encuesta, como de los resultados finales conseguidos tal como nos marca la ley de prevención de riesgos laborales (11) y el reglamento de los Servicios de Prevención (12) (13).

La encuesta, fue rellenada por los participantes en el tiempo de espera previo al reconocimiento médico y supervisada posteriormente por el médico del trabajo al iniciar la visita.

La recogida de los datos fue entre Marzo de 2006 y Junio de 2007 y, por tanto, siempre posterior a la entrada en vigor de la ley 28/2005, en enero de 2006. 
Nuestra plantilla total en las provincias participantes, era aproximadamente de 5.650 personas con las oscilaciones mensuales ligadas a la contratación de personal eventual. Han participado en la encuesta en estas provincias un total de 1432 personas, de las 1.680 que habían solicitado el reconocimiento periódico de la salud. No se realizó ningún tipo de selección previa, incluyendo tanto a personal funcionario, como laboral con contratos fijos o en situación de laboral eventual. Este tamaño muestral, nos permite obtener una seguridad del $99 \%$ y una precisión del $1 \%$ en los resultados. Las preguntas realizadas para la obtención de estos datos eran, tal como se adjunta: Centro de trabajo, Puesto de trabajo, Edad (en años), Sexo (mujer/hombre), Colectivo laboral al que pertenece (superior/gestión/ejecutivo/oficial postal/cartero/ayudante postal), ¿Actualmente usted fuma? (Sí, fumo diariamente/ Sí, fumo ocasionalmente/ No, no he fumado nunca/ No, soy un ex fumador/a).

Los resultados obtenidos se analizaron según el procedimiento estadístico de análisis descriptivo, donde los resultados se expresan como la media para variables cuantitativas y con proporciones para las variables cualitativas. En la comparación de medias se utilizó la prueba t de Student-Fisher, aplicándose la prueba no paramétrica correspondiente (test $\mathrm{U}$ de Mann-Whitney) en caso de no cumplirse el principio de normalidad. En la comparación de proporciones se utilizó la prueba de Pearson. En todos los cálculos se aceptó como nivel de significación un valor de $\mathrm{p}$ inferior a 0.05 . El análisis estadístico ha sido realizado con el programa SPSS v.14

\section{RESULTADOS}

De las 5.650 personas que componen la plantilla en las 5 provincias participantes, solicitaron reconocimiento de vigilancia periódica de la salud 1.680 $(29,73 \%$ del total de la plantilla de trabajadores/as) y entre estas, respondieron a la encuesta de tabaquismo 1.432 personas, siendo la tasa de respuestas obtenidas del 85,23\% de entre los que acudieron a los reconocimientos periódicos de vigilancia de la salud.

En cuanto a las características de la población laboral encuestada, el tamaño muestral fue de 1432 personas, la edad: media 44,09 años, en cuanto a diferencias por sexos: $56 \%$ hombres, $44 \%$ mujeres (FIGURA 1), el colectivo mayoritario fue el de ACR, correspondiente a auxiliares de clasificación y reparto (carteros/as) (FIGURA 2). Respecto al hábito tabáquico: No fumadores/as 39,1\%, Fumado-

\section{Figura 1. Distribución de los trabajadores por sexos}
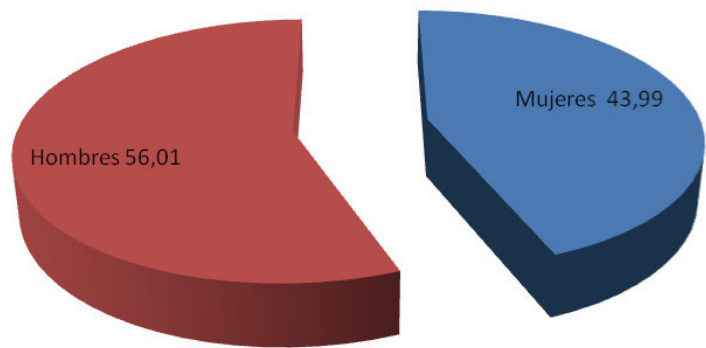

res diarios 29,9\%, Fumadores ocasionales 5,2\%, Ex fumadores/as 29,7\% (FIGURA 3). Estos resultados se muestran en la (Tabla 1)

Figura 2. Distribución de los trabajadores según grupo laboral de origen

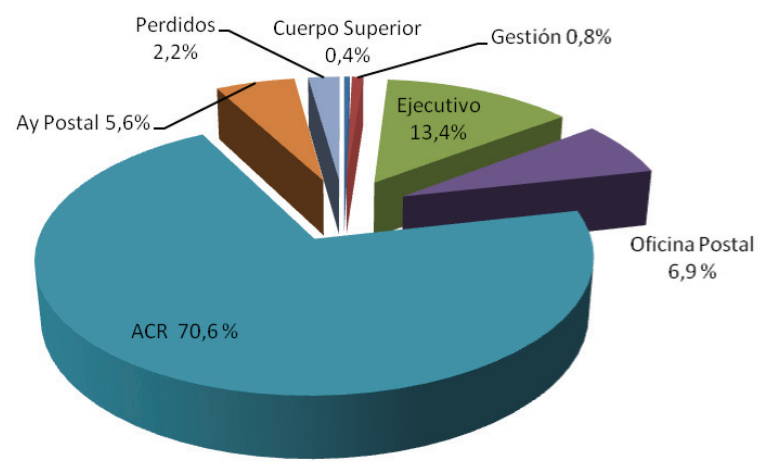

Figura 3. Distribución de los trabajadores por hábito tabáquico

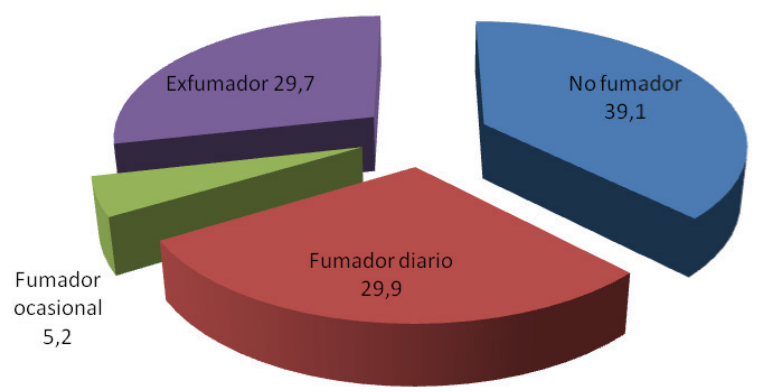

No ha habido variaciones geográficas en los resultados obtenidos. Existe una mayor cantidad de fumadores activos en puestos de trabajo con mayor cualificación lo que podría deberse a factores de riesgo como el estrés o el sedentarismo ligados a estos puestos de trabajo de mayor responsabilidad y 
Tabla 1. Características de la población laboral encuestada

\begin{tabular}{|c||l||l||c||}
\hline \multicolumn{1}{|c||}{ Opciones de respuesta } & \multicolumn{1}{|c||}{ \% Válido } & \multicolumn{1}{|c||}{ Media } \\
\hline \hline Edad & Mínimo 21 años & & $\begin{array}{c}44,09 \text { años } \\
\text { Desv. tip. 7,980 }\end{array}$ \\
& Máximo 64 años & & \\
\hline Sexo & Hombre & 56,0 & \\
& mujer & 44,0 & \\
\hline \hline $\begin{array}{c}\text { Colectivo } \\
\text { laboral }\end{array}$ & Cuerpo superior & 0,4 & \\
& Cuerpo de gestión & 0,8 & \\
& Cuerpo ejecutivo & 13,7 & \\
& Oficiales postales & 7,1 & \\
& Carteros/as & 72,2 & \\
& Ayudantes postales & 5,8 & \\
\hline \hline Hábito & Fuma diariamente & 25,9 & \\
tabáquico & Fuma ocasionalmente & 5,2 & \\
& Nunca fumador & 39,1 & \\
& Ex fumador & 29,7 & \\
\hline
\end{tabular}

Desv. tip. $=$ desviación típica

de carácter administrativo en su mayoría. No hay diferencias estadísticamente significativas, por sexos en los resultados obtenidos. Si hay diferencias respecto a la edad de los trabajadores fumadores con un mayor numero de fumadores activos entre el grupo de edad de 36-50 años. (Tabla 2)

Comparando los colectivos de trabajadores, fumadores activos con el de ex fumadores, vemos que la edad de los exfumadores es mas elevada que la de los fumadores, siendo los resultados estadísticamente significativos ( $\mathrm{p} \_$valor $<0,001$ ), las diferencias por sexo entre fumadores/as y exfumadores/as, no son estadísticamente significativas ( $\mathrm{p}$ valor $>$ $0,05)$. Existen diferencias estadísticas ligeramente significativas entre los dos grupos en función del puesto de trabajo desempeñado ( $p_{-}$valor $\left.=0,05\right)$ con una mayor proporción de fumadores/as en los grupos más cualificados o con puestos mas sedentarios, tales como el superior, que muestra además el menor numero de exfumadores. Existe también una elevada proporción de fumadores en el grupo de personal menos cualificado, de ayudantes postales, aunque con mayor número de exfumadores asociado que el que aparece en el cuerpo superior. También hay diferencias significativas ( $\mathrm{p}$ _valor 0,008 ) en el numero de cigarrillos/día consumidos, siendo mayor en el de los exfumadores/as, grupo este que ha comenzado a fumar mas tarde que en el de fumadores/as en activo, aunque las diferencias no son estadísticamente significativas ( $\mathrm{p}$-valor $>0,05$ ). El consumo de paquetes/año si es significativamente mayor entre el per- sonal fumador actualmente, que entre el ex fumador (p_valor $<0,05$ ) y por último también existen diferencias significativas en el número de años de fumador a favor de los fumadores/as en activo frente al grupo de ex fumadores/as ( $\left.p \_v a l o r<0,05\right)$. Los resultados, se muestran en la (Tabla 3)

\section{DISCUSIÓN Y CONCLUSIONES}

La tasa de participación en la encuesta de tabaquismo ha sido del $85,23 \%$ de la población laboral que accedió a la vigilancia periódica de la salud. Esta respuesta obtenida, ha sido más elevada que la conseguida en estudios anteriores en nuestra empresa, probablemente debido al método utilizado, durante los reconocimientos periódicos de vigilancia de la salud, momento en el que nos encontramos con una mayor receptibilidad a la participación por parte de los/as trabajadores/as. La tasa de respuestas había sido inferior, en encuestas previas realizadas en Correos con otros métodos de captación y por distintas patologías, así, en la encuesta de cefalea, que se realizó en 2002 acompañando a las nóminas, la tasa de respuesta fue del $16 \%$ y en la encuesta de asma laboral realizada en 2006 mediante un procedimiento mixto, durante los reconocimientos o con entrega a través de jefes/as o del personal sanitario, la tasa de respuestas fue aproximadamente del $45 \%$, en ambos casos, inferior a la tasa obtenida mediante el procedimiento actual que parece ser el mas efectivo y mejor acogido en nuestro ámbito laboral. 
Tabla 2. Relaciones entre el habito tabáquico de los/as trabajadores/as y distintos factores socio-laborales

\begin{tabular}{|c|c|c|c|c|c|c|}
\hline $\begin{array}{l}\text { Relación de } \\
\text { factores } \\
\text { estudiada }\end{array}$ & OPCIONES & $\begin{array}{c}\text { Fuma } \\
\text { Diariamente* }\end{array}$ & $\begin{array}{c}\text { Fuma } \\
\text { Ocasionalmente* }\end{array}$ & Nunca & $\begin{array}{c}\text { Ex } \\
\text { fumador* }\end{array}$ & p-Valor** \\
\hline $\begin{array}{l}\text { Tabaco y } \\
\text { centro de } \\
\text { trabajo }\end{array}$ & $\begin{array}{l}\text { Cádiz } \\
\text { Castellón } \\
\text { Gerona } \\
\text { Madrid } \\
\text { Valencia }\end{array}$ & $\begin{array}{r}24,0 \\
25,0 \\
24,0 \\
25,5 \\
27,0\end{array}$ & $\begin{array}{l}1,0 \\
4,2 \\
6,0 \\
6,4 \\
5,6\end{array}$ & $\begin{array}{r}36,5 \\
44,3 \\
45,2 \\
44,7 \\
35,8\end{array}$ & \begin{tabular}{r|}
38,5 \\
26,6 \\
24,8 \\
23,4 \\
31,6
\end{tabular} & $\begin{array}{l}\text { NS } \\
0,072>0,05\end{array}$ \\
\hline $\begin{array}{l}\text { Tabaco y } \\
\text { colectivo } \\
\text { laboral }\end{array}$ & $\begin{array}{l}\text { Superior } \\
\text { Gestión } \\
\text { Ejecutivo } \\
\text { O. postal } \\
\text { Cartero/a } \\
\text { Ayu. Postal }\end{array}$ & \begin{tabular}{|l|}
83,3 \\
33,3 \\
23,6 \\
27,7 \\
25,4 \\
30,5
\end{tabular} & $\begin{array}{l}0,0 \\
8,3 \\
4,1 \\
3,0 \\
5,3 \\
8,5\end{array}$ & $\begin{array}{l}0,0 \\
8,3 \\
40,0 \\
34,7 \\
39,0 \\
46,3\end{array}$ & \begin{tabular}{|l|}
16,7 \\
50,0 \\
32,3 \\
34,7 \\
30,0 \\
14,6
\end{tabular} & $0,014<0,05$ \\
\hline $\begin{array}{c}\text { Tabaco y } \\
\text { sexo }\end{array}$ & $\begin{array}{l}\text { Hombre } \\
\text { Mujer }\end{array}$ & $\begin{array}{l}26,4 \\
24,8\end{array}$ & $\begin{array}{l}4,4 \\
6,2\end{array}$ & $\begin{array}{l}37,5 \\
41,6\end{array}$ & $\begin{array}{l}31,7 \\
27,5\end{array}$ & $\mathrm{NS}>\mathbf{0 , 0 5}$ \\
\hline $\begin{array}{c}\text { Tabaco } y \\
\text { edad }\end{array}$ & $\begin{array}{l}\text { 20-35 años } \\
\text { 36-50 años } \\
51-65 \text { años }\end{array}$ & $\begin{array}{r}29,3 \\
27,9 \\
18,4\end{array}$ & $\begin{array}{l}4,5 \\
5,4 \\
5,0\end{array}$ & $\begin{array}{l}52,0 \\
35,1 \\
42,4\end{array}$ & $\begin{array}{l}14,1 \\
31,6 \\
34,1\end{array}$ & $<\mathbf{0 , 0 5}$ \\
\hline
\end{tabular}

* Valor numérico expresado en porcentaje

** Chi cuadrado de Pearson $\left(\chi^{2}\right) \mathrm{NS}=$ no significativo 
Tabla 3. Diferencias de los factores objeto del estudio entre los grupos de Fumadores/as y ex fumadores/as

\begin{tabular}{|c|c|c|c|c|}
\hline Factor de estudio & Opciones & Fumador & Ex fumador & P-Valor* \\
\hline Edad media & & 42,80 años & 45,64 años & $<0.001$ \\
\hline Sexo & $\begin{array}{l}\text { Hombre } \\
\text { Mujer }\end{array}$ & $\begin{array}{l}45,5 \% \\
47,4 \%\end{array}$ & & $\mathrm{NS}>0,05$ \\
\hline Puesto de trabajo & $\begin{array}{l}\text { C. Superior } \\
\text { C. Gestión } \\
\text { C. Ejecutivo } \\
\text { C. Of. postal } \\
\text { Cartero/a } \\
\text { C. Ayu. Postal }\end{array}$ & $\begin{array}{l}83,3 \% \\
40,0 \% \\
42,2 \% \\
44,4 \% \\
45,8 \% \\
67,6 \%\end{array}$ & \begin{tabular}{|l|}
$16,7 \%$ \\
$60,0 \%$ \\
$57,8 \%$ \\
$55,6 \%$ \\
$54,2 \%$ \\
$32,4 \%$
\end{tabular} & 0,05 \\
\hline$N^{0}$ cig/día & & 14,80 & 17,02 & 0,008 \\
\hline Edad inicio & & 18,47 años & 17,93 años & $\mathrm{NS}>0,05$ \\
\hline Paquetes/año & & 19,4061 & 17,5854 & $<0,05$ \\
\hline$N^{0}$ años de fumador & & 25,39 años & 19,10 años & $<0,05$ \\
\hline
\end{tabular}

La edad de los participantes en el estudio, coincide con la edad media de nuestra población laboral y en cuanto a las diferencias por sexos en los participantes, ha habido una mayor participación de hombres que de mujeres coincidiendo también con la mayor presencia de hombres entre nuestro personal trabajador en Correos.

En cuanto al grupo laboral de pertenencia de los trabajadores participantes en la encuesta de tabaquismo, la mayor participación ha correspondido al grupo laboral de carteros/as (Auxiliares de Clasificación y Reparto), que es también el grupo mayoritario en la empresa.

Entre los/as trabajadores/as encuestados/as, predomina el personal no-fumador, aunque, todavía sigue siendo elevada la cifra de fumadores/as en nuestra empresa $(35,1 \%)$, pese a las limitaciones en cuanto al consumo de tabaco que ha supuesto la entrada en vigor de la ley $28 / 2005$. Esta cifra es algo más elevada que la estimada en la encuesta nacional de salud 2003 que nos habla de cifras de prevalencia de consumo de tabaco del $31 \%$, aunque datos provisionales correspondientes a la ENS 2006 que muestran un descenso, que situaría la tasa de fumadores en el 29,95\% de población española.

La ubicación geográfica de los/as trabajadores/as, no influye en su habito tabáquico, por lo menos en las 5 provincias españolas consideradas en el estudio. Si que hemos apreciado diferencias significativas en relación al puesto de trabajo desempeñado, con un mayor número de fumadores entre los trabajadores del cuerpo superior, permitiéndonos pensar que la mayor cualificación profe- 
sional pudiera conllevar un mayor consumo de tabaco, posiblemente debido a factores favorecedores como el estrés o el sedentarismo ligado a la actividad laboral de estos puestos, aunque como limitación a estas conclusiones está el hecho de que en estos grupos de trabajo ha habido una menor participación de trabajadores en la encuesta y esto puede suponer un sesgo en los resultados. Sin embargo, estudios previos como el realizado por la asociación de ayuda contra la drogadicción en 1996, refuerzan estos resultados mostrando un mayor consumo de tabaco en el grupo de funcionarios y en trabajadores cualificados (14).

El sexo como hemos visto, no influye en el mayor hábito tabáquico, aunque si lo hace la edad con un mayor consumo en el bloque de edad de los 36-50 años. Sin embargo hemos de destacar la importancia especial del tabaquismo en las mujeres, tanto mas cuanto que afecta a las edades fértiles, con los posibles riesgos derivados para la gestación y para la descendencia.

Si estudiamos las co-relaciones entre los grupos de fumadores y ex fumadores y los factores antes analizados, vemos que en cuanto a la edad, son más jóvenes los fumadores que los ex-fumadores, sin influir el sexo en el hábito de forma significativa. Con respecto al grupo laboral de origen al que pertenecen los trabajadores, hay pocas diferencias estadísticas entre ambos grupos. Sin embargo, si que vemos diferencias significativas en su historial tabáquico analizando los dos grupos, así, en la cantidad de cigarrillos que fuman, existe un mayor consumo de cigarrillos/día entre los ex-fumadores que entre los fumadores en activo, los ex-fumadores comenzaron más jóvenes con el habito tabáquico, que los/as actuales fumadores/as en activo, aunque las diferencias entre ambos grupos no son lo suficientemente significativas y existe un mayor consumo de paquetes/año entre los fumadores/as en activo que entre los ex-fumadores/as. Los fumadores/as actualmente en activo, tienen mayor número de años de consumo que quienes ya han abandonado el habito tabáquico. Vemos por tanto que el perfil varía en ambos grupos, siendo la intensidad del consumo mayor entre los/as trabajadores/as fumadores/as actuales.

En España, según el Instituto Nacional de Estadística (INE 2005), la población activa ocupada la forman 18,9 millones de personas (el 66\% de los hombres y el $40 \%$ de las mujeres), por lo que es fácil entender que las intervenciones que se realizan en el entorno laboral consigan un gran impacto.
Según los resultados de un informe elaborado para el Comité Nacional de Prevención del Tabaquismo (4), cada trabajador que fuma, le cuesta a su empresa unos 1.500 euros al año en absentismo, pérdida de productividad y conservación y mantenimiento de instalaciones, una cifra que en toda España supone un gasto anual de 7.840 millones de euros. Además, si se cumplen las previsiones de este estudio, la prohibición de fumar en los centros de trabajo supondrá un importante ahorro para las empresas en los próximos años. Si bien durante este primer año, como ya hemos visto en nuestro estudio en Correos, el descenso del consumo de tabaco es todavía, relativamente modesto, la reducción del gasto de las empresas, a partir de la entrada en vigor de la ley 28/2005, podría llegar a ser del $29 \%$ a los diez años de su aplicación y, finalmente, en el año 2020, el descenso alcanzaría el 48\%”.

Dada la gravedad del problema del tabaquismo en la población general y específicamente la magnitud del consumo en la población activa, que podemos también observar en nuestro estudio en la población empleada en Correos, consideramos prioritario intensificar las actuaciones para la sensibilización acerca de la importancia de lograr espacios libres del humo de tabaco, incrementando simultáneamente las intervenciones de apoyo a la deshabituación en el marco de la salud laboral y midiendo su eficacia.

Es importante destacar, que el impacto del tabaco sobre la salud, no afecta sólo a las personas que fuman, sino también a quienes se ven involuntariamente expuestos/as al humo ambiental. El ambiente laboral ha sido, hasta la aprobación de la Ley $28 / 2005$, uno de los espacios en los que se producía con mayor intensidad el tabaquismo pasivo. Aun hoy, en el sector de hostelería, sigue siendo así en un alto porcentaje de locales.

Desde el comienzo de los años ochenta han aparecido estudios que muestran el mayor riesgo de cáncer de pulmón en personas expuestas al humo ambiental del tabaco (HAT). En 1986 el Surgeon General de Estados Unidos, publicó un informe, el que advertía de los riesgos del tabaquismo pasivo. Desde entonces numerosos estudios han ido confirmando esta evidencia, uno de los más recientes es el Informe de la Internacional Agency for Research on Cancer-IARC- de la OMS, 2004 (15), en el que se llega a establecer que la exposición al humo del tabaco en el lugar de trabajo produce un exceso de riesgo de cáncer de pulmón en un $20 \%$. Esto hizo que la IARC definiera al HAT como un cancerígeno 
humano del grupo 1. El estudio prospectivo EPIC 2005, así lo confirma. (16)

Existe además evidencia de la asociación entre el tabaquismo pasivo y las enfermedades respiratorias, enfermedad cardiovascular y agravamiento del asma. Muchos estudios vienen demostrando desde mediados de los noventa la asociación del tabaquismo pasivo con la enfermedad cardiaca isquémica, encontrando excesos de riesgo de hasta el 25\%. (17)

La eliminación a la exposición al humo del tabaco (HAT) en el medio laboral tiene una clara repercusión sobre el nivel de salud. En los Estados Unidos han estimado que entre el 5 al $15 \%$ de los cánceres de pulmón y un gran número de infartos en personas que nunca han fumado podrían evitarse eliminando el HAT. (17)

Tenemos pues suficiente evidencia científica para saber la importancia del tabaco como problema de salud, justifica sobradamente estas intervenciones que además se apoyan en políticas de salud como la Ley 28/2005 o la Ley de Prevención de Riesgos Laborales 31/95. Existen además, como hemos comentado brevemente y como muestran distintos estudios, razones económicas que refuerzan los beneficios que pueden obtenerse de los programas de apoyo a la deshabituación y las estrategias de espacios libres de humo. (4)

Los costes económicos derivados de los problemas de salud en personas fumadoras se deben principalmente, al aumento de bajas por enfermedad, la mortalidad prematura, la contratación de personal de sustitución para cubrir bajas, la pérdida de productividad, el incremento de los gastos de explotación o el incremento de las jubilaciones anticipadas, se estima que aproximadamente $2 / 3$ de estas jubilaciones se deben al tabaquismo.
Pero además la exposición al HAT en el medio laboral produce problemas de salud en personas que nunca han fumado, aumentando la incidencia de algunas enfermedades, (sobre todo respiratorias y cardiovasculares) con sus consecuentes efectos sobre la productividad, el absentismo, las bajas, etc. (17) (18) (19)

Vemos pues, que desde el mundo del trabajo nos queda todavía mucho por hacer en nuestra empresa, dada la elevada cifra de fumadores en activo que persisten en el momento actual, con datos que pueden extrapolarse a otras empresas del ámbito publico o privado y en el que la puesta en vigor de la ley $28 / 2005$ no ha supuesto mas que el comienzo de un largo camino por recorrer en el que la actuación del personal sanitario laboral, tanto médicos/as como enfermeros/as, con la activación, desde los servicios de prevención de unidades de deshabituación tabáquica para el que es imprescindible recibir el apoyo de los órganos directivos de las empresas y de la sanidad publica en España. Intensificar y mejorar nuestras estrategias de intervención para facilitar la deshabituación, nos permitirá en un futuro comprobar la efectividad de nuestras actuaciones con las que podemos conseguir la disminución de las cifras de consumo de tabaco entre nuestros trabajadores/as y los consiguientes beneficios que esto comporta, tanto en mejoras de salud, con la reducción de la morbi-mortalidad, como por los beneficios económicos subsiguientes para las empresas y para la sociedad.

\section{AGRADECIMIENTOS}

Dirección de RRHH de Correos, Laura Carrera, José Benaches, Manuel-Luís Gomar López y Purificación Martínez (enfermeros/as de Correos), $\mathrm{M}^{\mathrm{a}}$ José Peña y Amparo Amorós (Auxiliares de Correos), Rafael Martínez Esteve (traductor). 


\section{BIBLIOGRAFIA}

1. Isabel Saiz Martínez-Acitores et al. Plan Nacional de Control y Prevención del tabaquismo. Rev. Esp. Salud Pública 2003; 77:441-473.

2. Ministerio de Sanidad y consumo. Instituto Nacional de estadística. Encuesta nacional de Salud de España 2006.

3. J González-Enríquez et al. Morbilidad, mortalidad y costes sanitarios evitables mediante una estrategia de tratamiento del tabaquismo en España. Gac. Sanit. 2002; 16: 308 - 317

4. Ángel López Nicolás, Jaime Pinilla Domínguez. Evaluación del impacto de la Ley de Medidas Sanitarias Contra el Tabaquismo en el sector hostelero: bares y restaurantes. 2006 Informe CNPT

5. Ley $28 / 2005$, de 26 de diciembre, de medidas sanitarias frente al tabaquismo y reguladora de la venta, el suministro, el consumo y la publicidad de los productos del tabaco.

6. AA López. Guía práctica para la implantación de unidades de deshabituación tabáquica desde los Servicios de Prevención, enfermería y otras unidades sanitarias. Editorial Médica Internacional; 2004. ISBN: 84-9627706-2.

7. AA López. Guía para un abordaje integral del tabaquismo en la empresa. Editada por la Conselleria de Salut i Consum y la Conselleria de Treball i Formació del Govern de les Illes Balears; 2005.

8. Ministerio de sanidad. Comité Nacional para la Prevención del Tabaquismo. Madrid. 2005. Se puede dejar de fumar. Claves para conseguirlo.

9. Comunidad de Madrid. Consejería de Sanidad y Consumo. Dirección General de Salud Pública, Alimentación y Consumo Ayuntamiento de Madrid. Concejalía de Gobierno de Seguridad y Servicios a la Comunidad. Ed. Instituto de Salud Pública. Madrid 2004. Guía de apoyo para el abordaje del tabaquismo

10. ENS 2003 Ministerio de Sanidad, en www.msc.es
11. LEY 31/1995, de 8 de noviembre de prevención de riesgos laborales. BOE $\mathrm{n}^{\circ} 269$, de 10 de noviembre. http://www.mtas.es/insht/legislation/L/ lprl.htm

12. REAL DECRETO 39/1997, de 17 de enero, por el que se aprueba el Reglamento de los Servicios de Prevención. BOE núm. 27 de 31 enero. http://www.mtas.es/insht/legislation/RD/rd39.htm

13. Real Decreto 780/1998, de 30 de abril, por el que se modifica el Real Decreto 39/1997, de 17 de enero, por el que se aprueba el Reglamento de los servicios de prevención. http://www.mtas.es/insht/ legislation/RD/rd39.htm

14. Fundación de ayuda contra la drogadicción. La Incidencia de las drogas en el mundo laboral.1996.

15. International Agency for Research a Cancer. Tobacco Smoker and involuntary smoking IARC. Monographs on the Evaluation of carcinogenic risks to humans. Vol. 38. Lyon; IARC; 2004.

16. Vineis $\mathrm{P}$ et al. Environmental tobacco smokers risk of respiratory cancer and chronic obstructive pulmonary disease in former smokers and never smokers in the EPIV prospective study. BMJ 2005; $330: 277-80$.

17. Comité Nacional de Prevención del Tabaquismo. Ministerio de Sanidad. Espacios libres de humo. Revisión de la evidencia científica sobre el impacto sanitario y económico de la regulación del humo ambiental del tabaco en el medio laboral.2006

18. Kaur $\mathrm{S}$ et al The impact of environmental tobacco on women's risk of dying from Herat disease: a meta-analysis. J Women's Health. 2004; 13:888-97.

19. González-Alonso, Julia et al El reto del control el tabaquismo en el lugar de trabajo. Revista Española de Salud Pública, 2003; 77(1):1-2 